Y. Ikeda

Nagoya Math. J.

Vol. 60 (1976), 151-172

\title{
REGULARITY OF SOLUTIONS FOR QUASI-LINEAR PARABOLIC EQUATIONS
}

\author{
YOSHIAKI IKEDA
}

\section{§ 1. Introduction.}

Let $\Omega$ be a bounded domain in $n$-dimensional Euclidian space $E^{n}$ ( $n \geqq 2$ ), and consider the space-time cylinder $Q=\Omega \times(0, T]$ for some fixed $T>0$. In this paper we deal with the Cauchy and Dirichlet problem for a second order quasi-linear equation

$$
\begin{array}{r}
u_{t}-\operatorname{div} \mathscr{A}\left(x, t, u, u_{x}\right)+B\left(x, t, u, u_{x}\right)=0 \quad \text { for }(x, t) \in Q, \\
u(x, 0)=\phi(x) \text { in } \Omega \text { and } u(x, t)=\psi(x, t) \\
\quad \text { for }(x, t) \in \Gamma=\partial \Omega \times(0, T],
\end{array}
$$

where $\partial \Omega$ is a boundary of $\Omega$ which satisfies the following condition $(A)$ : Condition $(A)$. There exist constants $\rho_{0}$ and $\lambda_{0}$ both in $(0,1)$ such that, for any sphere $K(\rho)$ with center on $\partial \Omega$ and radius $\rho \leqq \rho_{0}$, the inequality meas $[K(\rho) \cap \Omega] \leqq\left(1-\lambda_{0}\right) \times$ meas $K(\rho)$ holds, where meas $E$ means the measure of a measurable set $E$.

In the equation $\mathscr{A}=\left(\mathscr{A}_{1}, \cdots, \mathscr{A}_{n}\right)$ is a given vector function of $\left(x, t, u, u_{x}\right)$, $B$ is a given scalar function of the same variables, and $u_{x}=\left(\frac{\partial u}{\partial x_{1}}, \cdots, \frac{\partial u}{\partial x_{n}}\right)$ denotes the spatial gradient of the dependent variable $u=u(x, t)$. Also $\operatorname{div} \mathscr{A}$ refers to the divergence of the vector $\mathscr{A}\left(x, t, u, u_{x}\right)$ with respect to the variables $x=x\left(x_{1}, \cdots, x_{n}\right)$. The functions $\phi(x)$ and $\psi(x, t)$ in (1.2) are bounded, measurable and belong to the spaces $L^{2}(\Omega)$ and $L^{\infty}\left[0, T ; L^{2}(\tilde{\Omega})\right]$ $\cap L^{\alpha}\left[0, T ; H^{1, \alpha}(\tilde{\Omega})\right]$ respectively, where $\tilde{\Omega}$ is a domain containing $\Omega$.

Throughout the paper we assume that $\mathscr{A}$ and $B$ satisfy inequalities of the form

$$
\left\{\begin{array}{l}
p \cdot \mathscr{A}(x, t, u, p) \geqq a_{0}|p|^{\alpha}-c(x, t)|u|^{\alpha}-f(x, t) \\
|B(x, t, u, p)| \leqq b(x, t)|p|^{\alpha-1}+d(x, t)|u|^{\alpha-1}+g(x, t), \\
|\mathscr{A}(x, t, u, p)| \leqq \bar{a}|p|^{\alpha-1}+e(x, t)|u|^{\alpha-1}+h(x, t),
\end{array}\right.
$$

Received November 18, 1974. 
for any $n$-dimensional real vector $p$ and for any real number $\alpha>2$. Here $a_{0}$ and $\bar{a}$ are positive constants and the coefficients $b, c, d, e, f, g, h$ are non-negative functions of $(x, t)$ and $b^{\alpha}, c, d, e^{\alpha /(\alpha-1)}, f, g, h^{\alpha /(\alpha-1)}$ belong to some space $L^{p, q}(Q)$, where $p$ and $q$ are non-negative real numbers satisfying

$$
\frac{n}{\alpha p}+\frac{\alpha n-2 n+2 \alpha}{2 \alpha q}<1 \quad \text { when } \alpha<n
$$

and

$$
\frac{1}{p}+\frac{\alpha}{2 q}<1-\varepsilon_{0}, \quad p>1
$$

for any sufficiently small $\varepsilon_{0}>0$ when $\alpha \geqq n$.

A function $w=w(x, t)$ which is measurable on $Q$ will be said to belong to the class $L^{p, q}(Q)$ if the iterated integral

$$
\|w\|_{p, q}=\left\{\int_{0}^{T}\left(\int_{\Omega}|w|^{p} d x\right)^{q / p} d t\right\}^{1 / q}
$$

is finite. If a function $w(x)$ which is measurable on $\Omega$ possesses a distribution derivative $\left(u_{x_{1}}, \cdots, u_{x_{n}}\right)$ and if $\|w\|_{L^{p}(\Omega)}+\left\|w_{x}\right\|_{L^{p}(\Omega)}<\infty$, then $w(x)$ is said to belong to $H^{1, p}(\Omega)$, where $\left\|w_{x}\right\|_{L^{p}(\Omega)}^{p}=\sum_{i=1}^{n}\left\|u_{x_{i}}\right\|_{L^{p}(\Omega)}^{p}$.

The space $H_{0}^{1, p}(\Omega)$ is the completion of $C_{0}^{\infty}(\Omega)$ with respect to this norm.

We denote by $L^{q}\left[0, T ; H^{1, p}(\Omega)\right]$ the space of functions $w(x, t)$ with the following properties:

(i) $w(x, t)$ is measurable on $Q$,

(ii) for almost all $t \in(0, T], w(x, t) \in H^{1, p}(\Omega)$,

(iii) $\|w(x, t)\|_{H_{1, p(\Omega)}} \in L^{q}[0, T]$.

The function $u$ is said to be a weak solution of the problem (1.1), (1.2) if $u$ belongs to the space $H^{1,2}\left[0, T ; L^{2}(\Omega)\right] \cap L^{\infty}\left[0, T ; L^{2}(\Omega)\right] \cap$ $L^{\alpha}\left[0, T ; H^{1, \alpha}(\Omega)\right]$ and if $u$ satisfies the following conditions:

$$
\int_{t_{0}}^{t_{1}} \int_{\Omega}\left\{u_{t} \Phi(x, t)+\mathscr{A}\left(x, t, u, u_{x}\right) \Phi_{x}+B\left(x, t, u, u_{x}\right) \Phi\right\} d x d t=0
$$

for any $t_{0}, t_{1}\left(0 \leqq t_{0}<t_{1} \leqq T\right)$ and

$$
\lim _{t \rightarrow 0} \int_{\Omega} u(x, t) \Phi(x, t) d x=\int_{\Omega} \phi(x) \Phi(x, 0) d x
$$


for any continuously differentiable function $\Phi=\Phi(x, t)$ with compact support in $\Omega$. That the boundary value of $u$ is equal to $\psi(x, t)$ on $\Gamma$ in (1.2) means that $u(x, t)-\psi(x, T) \in L^{\infty}\left[0, T ; L^{2}(\Omega)\right] \cap L^{\alpha}\left[0, T ; H_{0}^{1, \alpha}(\Omega)\right]$ for $\psi(x, t) \in L^{\infty}\left[0, T ; L^{2}(\tilde{\Omega})\right] \cap L^{\alpha}\left[0, T ; H^{1, \alpha}(\tilde{\Omega})\right]$, where $\tilde{\Omega} \supset \bar{\Omega}$.

In section 4 we shall prove the boundedness of the solution of the problem (1.1), (1.2) when $\phi(x)$ and $\psi(x, t)$ are bounded. The same result was obtained by D. G. Aronson and J. Serrin [2] for non-linear parabolic equation (1.1) under the condition

$$
\left\{\begin{array}{l}
p \cdot \mathscr{A}(x, t, u, p) \geqq a|p|^{\alpha}-e^{\alpha}|u|^{\alpha}-h^{\alpha} \\
|B(x, t, u, p)| \leqq b|p|^{\alpha-1}+d^{\alpha-1}|u|^{\alpha-1}+g^{\alpha-1}
\end{array}\right.
$$

where coefficients $a, b, \cdots, g$ are non-negative constants.

In section 5 our main theorem states that if $u$ is a weak solution of the problem (1.1), (1.2), then $u$ is Hölder continuous in $Q$ and that, moreover if the boundary value $\psi(x, t)$ of $u$ is Hölder continuous then $u$ is Hölder continuous on $\bar{Q}=\bar{\Omega} \times(0, T]$.

This result extends theorems proved by Ladyzenskaya and Uralceva [3] on some linear and quasi-linear parabolic equations, theorems proved by Serrin [4] on quasi-linear elliptic equations, and those given by Aronson and Serrin [1] on the quasi-linear parabolic equations

$$
u_{t}=\operatorname{div} \mathscr{A}\left(x, t, u, u_{x}\right)+B\left(x, t, u, u_{x}\right)
$$

under the conditions

$$
\left\{\begin{array}{l}
p \cdot \mathscr{A}(x, t, u, p) \geqq a|p|^{2}-c^{2}|u|^{2}-f^{2}, \\
|B(x, t, u, p)| \leqq b|p|+d|u|+g, \\
|\mathscr{A}(x, t, u, p)| \leqq \bar{a}|p|+e|u|+h,
\end{array}\right.
$$

where $a$ and $\bar{a}$ are positive constants, while the coefficients $b, c, \cdots, h$ are non-negative functions of $(x, t)$ and each coefficient is contained in some space $L^{p, q}(Q)$, where

$$
p>2 \text { and } \frac{n}{2 p}+\frac{1}{q}<\frac{1}{2} \text { for } b, c, e, f, h
$$

and

$$
p>1 \text { and } \frac{n}{2 p}+\frac{1}{p}<1 \text { for } d, g .
$$




\section{§ 2. Preliminaries.}

In this section we shall state and prove several lemmas which are often used later.

Using the Hölder's inequality we can easily prove the following lemma :

LEMMA 2.1 (Aronson-Serrin [1]). If $w$ is contained in $L^{q, q_{1}} \cap L^{r, r_{1}}$, then $w$ is contained in $L^{p, p_{1}}$, where

$$
\frac{1}{p}=\frac{\lambda}{q}+\frac{\mu}{r}, \frac{1}{p_{1}}=\frac{\lambda}{q_{1}}+\frac{\mu}{r_{1}} \quad(\lambda, \mu \geqq 0, \lambda+\mu=1) .
$$

Moreover

$$
\|w\|_{p, p_{1}} \leqq\|w\|_{q, q_{1}}^{\lambda} \cdot\|w\|_{r, r_{1}}^{\mu}
$$

where

$$
\|w\|_{p, q}=\left(\int_{0}^{T}\left(\int_{\Omega}|w|^{p} d x\right)^{q / p} d t\right)^{1 / q}
$$

LEMMA 2.2 (Aronson-Serrin [1]). Let $w$ belong to the space $L^{\alpha}\left[0, T ; H_{0}^{1, \alpha}(\Omega)\right]$. Then

$$
\|w\|_{\alpha^{*}, \alpha} \leqq K\left\|w_{x}\right\|_{\alpha, \alpha},
$$

where $\frac{1}{\alpha^{*}}=\frac{1}{\alpha}-\frac{1}{n}$ when $n>\alpha$, and $\alpha^{*}$ is any finite number when $\alpha \geqq n$. The constant $K$ depends only on $\alpha, n$ and $\Omega$. If $n \leqq \alpha$, then $K$ depends on the choice of $\alpha^{*}$.

LEMMA 2.3. If $w$ belongs to the space $L^{\infty}\left[0, T ; L^{2}(\Omega)\right] \cap L^{\alpha}\left[0, T ; H_{0}^{1, \alpha}(\Omega)\right]$, then $w$ belongs to the space $L^{\alpha p^{\prime}, \alpha q^{\prime}}$ for all exponents pairs $\left(p^{\prime}, q^{\prime}\right)$ whose Hölder conjugate $(p, q)$ satisfies

$$
\frac{n}{\alpha p}+\frac{\alpha n-2 n+2 \alpha}{2 \alpha q}<1 \quad \text { when } \alpha<n
$$

and

$\frac{1}{p}+\frac{\alpha}{2 q}<1-\varepsilon_{0}$ for any sufficiently small $\varepsilon_{0}>0$ when $\alpha \geqq n$.

Moreover

$$
\|w\|_{\alpha p^{\prime}, \alpha q^{\prime}}^{\alpha} \leqq K T^{\nu}\left\{\|w\|_{2, \infty}^{\alpha}+\left\|w_{x}\right\|_{\alpha, \alpha}^{\alpha}\right\}
$$


and for any $\varepsilon>0$

$$
\|w\|_{\alpha p^{\prime}, \alpha q^{\prime}}^{\alpha} \leqq \varepsilon\left\|w_{x}\right\|_{\alpha, \alpha}^{\alpha}+C(\varepsilon) T^{\nu \alpha /(\alpha-1)}\|w\|_{2, \infty}^{\alpha},
$$

where $\nu=\left(1-\frac{1}{\kappa}\right) \frac{1}{q^{\prime}}, \kappa=\frac{2}{p^{\prime}}-\frac{2}{\alpha^{*} q^{\prime}}+\frac{1}{q^{\prime}}>1, K$ depends only on $\alpha, n$ and meas $\Omega$, and $C(\varepsilon)$ depends only on $\varepsilon, \alpha, n$ and meas $\Omega$.

Proof. Let $\kappa$ be a real number $>1$. Then by Hölder's inequality and Lemma 2.1,

$$
\|w\|_{\alpha p^{\prime}, \alpha q^{\prime}}^{\alpha} \leqq\|w\|_{\alpha p^{\prime} x, \alpha q^{\prime} \alpha}^{\alpha}\left\{T^{1 / q^{\prime}}(\operatorname{meas} \Omega)^{1 / p^{\prime}}\right\}^{1-1 / \kappa}
$$

and

$$
\|w\|_{\alpha p^{\prime} \kappa, \alpha q^{\prime} \kappa}^{\alpha} \leqq\|w\|_{\alpha^{*}, \alpha}^{\alpha}\|w\|_{2, \infty}^{\alpha-\lambda}
$$

provided that

$$
0 \leqq \lambda \leqq \alpha \text { and } \frac{1}{\kappa p^{\prime}}=\frac{\lambda}{\alpha^{*}}+\frac{\alpha-\lambda}{2}, \frac{1}{\kappa q^{\prime}}=\frac{\lambda}{\alpha} .
$$

These relations imply

$$
\lambda=\frac{\alpha}{\kappa q^{\prime}}, \quad \kappa=\frac{2}{\alpha p^{\prime}}-\frac{2}{\alpha^{*} q^{\prime}}+\frac{1}{q^{\prime}}>1 .
$$

From Young's inequality and Lemma 2.2 we have (2.1) and (2.2).

LEMMA 2.4. If the function $u(x)$ belongs to the space $H_{0}^{1, \alpha}(\Omega)$, then it holds

$$
\int_{\Omega}|u|^{\alpha} d x \leqq K \int_{\Omega}\left|u_{x}\right|^{\alpha} d x \cdot[\operatorname{meas} \Omega]^{\alpha / n}
$$

Proof. By Hölder's inequality, it is clear that

$$
\int_{\Omega}|u|^{\alpha} d x \leqq\left(\int_{\Omega}|u|^{\alpha^{*}} d x\right)^{\alpha / \alpha^{*}} \cdot(\operatorname{meas} \Omega)^{1-\alpha / \alpha^{*}}
$$

where $\alpha^{*}=\frac{n-\alpha}{\alpha n}$ when $\alpha<n$ and when $\alpha \geqq n, \alpha^{*}$ is any number $>\alpha$.

If $\alpha<n$, then $1-\frac{\alpha}{\alpha^{*}}=\frac{\alpha}{n}$ and from Sobolev's lemma we have our lemma.

If $\alpha \geqq n$, we take $\beta<n$ such that $\beta^{*}=\frac{n-\beta}{\beta n}=\alpha^{*}$. Then 


$$
\begin{aligned}
\left(\int_{\Omega}|u|^{\alpha^{*}} d x\right)^{\alpha / \alpha^{*}} \cdot(\operatorname{meas} \Omega)^{1-\alpha / \alpha^{*}} & \leqq K\left(\int_{\Omega}\left|u_{x}\right|^{\beta} d x\right)^{\alpha / \beta}(\operatorname{meas} \Omega)^{1-\alpha / \beta^{*}} \\
& \leqq K\left(\int_{\Omega}\left|u_{x}\right|^{\alpha} d x\right)(\operatorname{meas} \Omega)^{\alpha / \beta(1-\beta / \alpha)+1-\alpha / \beta^{*}} \\
& =K\left(\int_{\Omega}\left|u_{x}\right|^{\alpha} d x\right)(\operatorname{meas} \Omega)^{\alpha / n}
\end{aligned}
$$

\section{§ 3. Fundamental inequalities.}

In this section we shall derive some fundamental inequalities for weak solutions of the problem (1.1), (1.2), which are used in the following sections.

Let $u$ be a weak solution of the problem (1.1), (1.2) and for a real number $k$, put

$$
A_{k}(t)=\{x \in \Omega \mid u(x, t) \geqq k\} \quad \text { and } \quad B_{k}(t)=\{x \in \Omega \mid u(x, t)<k\} .
$$

We assume that the boundary value $\psi(x, t)$ and the initial value $\phi(x)$ belong to the spaces $L^{\infty}\left[0, T ; L^{2}(\Omega)\right] \cap L^{\alpha}\left[0, T ; H^{1, \alpha}(\Omega)\right]$ and $L^{2}(\Omega)$ respectively and they are bounded, i.e. there exists a positive constant $M_{0}$ such that

$$
|\psi(x, t)| \leqq M_{0}, \quad|\phi(x)| \leqq M_{0} .
$$

We put $M=\max _{0 \leq t \leq T}\left(\int_{\Omega} u^{2} d x\right)^{1 / 2}=\|u\|_{2, \infty}$, and $U=\frac{u}{M}$.

Then, since $u$ is a weak solution of (1.1), we have

$$
U_{t}-\frac{1}{M} \operatorname{div} \mathscr{A}\left(x, t, M U, M U_{x}\right)+\frac{1}{M} B\left(x, t, M U, M U_{x}\right)=0 .
$$

Thus, it holds that

$$
\begin{aligned}
\int_{t_{0}}^{t_{1}} \int_{\Omega}\left\{U_{t} \Phi+\frac{1}{M} \mathscr{A}\left(x, t, M U, M U_{x}\right) \Phi_{x}\right. \\
\left.+\frac{1}{M} B\left(x, t, M U, M U_{x}\right) \Phi\right\} d x d t=0
\end{aligned}
$$

for any differentiable function $\Phi(x, t)$ with compact support in $\Omega$.

It is clear that (3.3) is valid for $\Phi \in L^{\infty}\left[0, T ; L^{2}(\Omega)\right] \cap L^{\alpha}\left[0, T ; H_{0}^{1, \alpha}(\Omega)\right]$.

Now we put $u^{(k)}=\max (u, k)-k$.

If $k \geqq M_{0}$, then $u^{(k)} \in L^{\infty}\left[0, T ; L^{2}(\Omega)\right] \cap L^{\alpha}\left[0, T ; H_{0}^{1, \alpha}(\Omega)\right]$. Hence, taking $\Phi=u^{(k)}$ in (3.3), we have 


$$
\int_{t_{0}}^{t_{1}} \int_{A_{k}(t)}\left(U_{t} u^{(k)}+\frac{1}{M} \mathscr{A} \cdot u_{x}^{(k)}+\frac{1}{M} B \cdot u^{(k)}\right) d x d t=0 .
$$

If we put $U^{(k)}=\frac{u^{(k)}}{M}$, then, letting $t_{0} \rightarrow 0$, we see,

$$
\begin{gathered}
\int_{t_{0}}^{t_{1}} \int_{A_{k}(t)} U_{t} u^{(k)} d x d t=M \int_{t_{0}}^{t_{1}} \int_{A_{k}(t)} \frac{1}{2} \frac{\partial}{\partial t}\left\{\left(U^{(k)}\right)^{2}\right\} d x d t \\
\longrightarrow \frac{M}{2} \int_{A_{k}(t)}\left(U^{(k)}\right)^{2} d x \quad \text { as } t_{0} \rightarrow 0,
\end{gathered}
$$

because of $U^{(k)}(x, 0)=0$.

It is obvious from the condition (1.3) that

$$
\begin{aligned}
\int_{0}^{t_{1}} \int_{A_{k}(t)} & \frac{1}{M} \mathscr{A} \cdot u_{x}^{(k)} d x d t=\int_{0}^{t_{1}} \int_{A_{k}(t)} \mathscr{A} \cdot U_{x}^{(k)} d x d t \\
\geqq & \frac{a_{0}}{M} \int_{0}^{t_{1}} \int_{A_{k}(t)} M^{\alpha}\left|U_{x}^{(k)}\right|^{\alpha} d x d t-\frac{1}{M} \int_{0}^{t_{1}} \int_{A_{k}(t)} c(x, t)|M U|^{\alpha} d x d t \\
& \quad-\frac{1}{M} \int_{0}^{t_{1}} \int_{A_{k}(t)} f(x, t) d x d t
\end{aligned}
$$

and

$$
\begin{aligned}
& \int_{0}^{t_{1}} \int_{A_{k}(t)} \frac{1}{M} B \cdot u^{(k)} d x d t=\int_{0}^{t_{1}} \int_{A_{k}(t)} B U^{(k)} d x d t \\
& \leqq \int_{0}^{t_{1}} \int_{A_{k}(t)}\left\{b(x, t) M^{\alpha-1}\left|U_{x}^{(k)}\right|^{\alpha-1}\left|U^{(k)}\right|+d(x, t) M^{\alpha-1}|U|^{\alpha-1}\left|U^{(k)}\right|\right. \\
& \left.+g(x, t)\left|U^{(k)}\right|\right\} d x d t .
\end{aligned}
$$

Thus we obtain

$$
\begin{aligned}
& \frac{M}{2}\left\|U^{(k)}\right\|_{2, \infty}^{2}+a_{0} M^{\alpha-1}\left\|U_{x}^{(k)}\right\|_{\alpha, \alpha}^{\alpha} \\
& \leqq \int_{0}^{t_{1}} \int_{A_{k}(t)}\left\{M^{\alpha-1} b\left|U_{x}^{(k)}\right|^{\alpha-1}\left|U^{(k)}\right|+c M^{\alpha-1}|U|^{\alpha}+d M^{\alpha-1}\left|U^{\alpha-1}\right|\left|U^{(k)}\right|\right. \\
& \left.+\frac{1}{M} f+g\left|U^{(k)}\right|\right\} d x d t,
\end{aligned}
$$

where $\left\|U^{(k)}\right\|_{2, \infty}^{2}=\max _{0 \leqq t \leqq t_{1}} \int_{A_{k}(t)}\left(u^{(k)}\right)^{2} d x$

and

$$
\left\|U_{x}^{(k)}\right\|_{\alpha, \alpha}^{\alpha}=\int_{0}^{t_{1}} \int_{A_{k}(t)}\left|U_{x}^{(k)}\right|^{\alpha} d x d t
$$


Using Young's inequality, we see

$$
M^{\alpha-1} b\left|U_{x}^{(k)}\right|^{\alpha-1}\left|U^{(k)}\right| \leqq \frac{1}{2} a_{0} M^{\alpha-1}\left|U_{x}^{(k)}\right|^{\alpha}+C_{0} b^{\alpha} M^{\alpha-1}\left|U^{(k)}\right|^{\alpha}
$$

and

$$
M^{\alpha-1} d\left|U^{\alpha-1}\right|\left|U^{(k)}\right| \leqq C_{1} M^{\alpha-1} d\left\{|U|^{\alpha}+\left|U^{(k)}\right|^{\alpha}\right\}
$$

where $C_{0}$ and $C_{1}$ are positive constants depending only on $a_{0}$ and $\alpha$.

Since $U=U^{(k)}+\frac{k}{M}$ in $A_{k}(t)$, it follows that

$$
|U|^{\alpha} \leqq C_{2}\left\{\left|U^{(k)}\right|^{\alpha}+\left(\frac{k}{M}\right)^{\alpha}\right\}
$$

where $C_{2}$ is a positive constant depending only on $\alpha$.

Moreover, since $\left\|U^{(k)}\right\|_{2, \infty} \leqq 1$, it is clear that

$$
\left\|U^{(k)}\right\|_{2, \infty}^{\alpha} \leqq\left\|U^{(k)}\right\|_{2, \infty}^{2} \text {. }
$$

Thus we have from (3.5) (3.9),

$$
\begin{array}{r}
a_{1}\left(\left\|U^{(k)}\right\|_{2, \infty}^{\alpha}+\left\|U_{x}^{(k)}\right\|_{\alpha, \alpha}^{\alpha}\right. \\
\leqq C\left\{\int _ { 0 } ^ { t _ { 1 } } \int _ { A _ { k } ( t ) } \left\{\left(b^{\alpha}+c+d+1\right)\left|U^{(k)}\right|^{\alpha}+\left(1+k^{\alpha}\right)(c+d+f)\right.\right. \\
\left.+g\left|U^{(k)}\right|\right\} d x d t
\end{array}
$$

where $a_{1}=\min \left(\frac{M}{2}, \frac{a_{0}}{2} M^{\alpha-1}\right)$ and $C$ is a positive constant depending only on $\alpha$ and $M$.

If we put $\theta_{1}=b^{\alpha}+c+d+1$, then $\theta_{1}$ belongs to the space $L^{p, q}(Q)$ with $p$ and $q$ satisfying the inequality (1.5). Thus from Lemma 2.3, we see

$$
\begin{aligned}
& \int_{0}^{t_{1}} \int_{A_{k}(t)} \theta_{1}\left|U^{(k)}\right|^{\alpha} d x d t \\
& \quad \leqq\left\|\theta_{1}\right\|_{p, q}\left\|U^{(k)}\right\|_{\alpha p^{\prime}, \alpha q^{\prime}}^{\alpha} \\
& \left.\quad \leqq K\left\|\theta_{1}\right\|_{p, q} t_{1}^{\gamma}\left\|U_{x}^{(k)}\right\|_{\alpha, \alpha}^{\alpha}+\left\|U^{(k)}\right\|_{2, \infty}^{\alpha}\right) .
\end{aligned}
$$

Similarly if we put $\theta_{2}=c+d+f$, then $\theta_{2} \in L^{p, q}$. Thus we see

$$
\begin{aligned}
& \int_{0}^{t_{1}} \int_{A_{k}(t)} \theta_{2}\left(1+k^{\alpha}\right) d x d t \\
& \quad \leqq\left(1+k^{\alpha}\right)\left\|\theta_{2}\right\|_{p, q}\left(\int_{0}^{t_{1}}\left(\operatorname{meas} A_{k}(t)\right)^{q^{\prime} / p^{\prime}} d t\right)^{1 / q^{\prime}},
\end{aligned}
$$


and

$$
\begin{aligned}
& \int_{0}^{t_{1}} \int_{A_{k}(t)} g\left|U^{(k)}\right| d x d t \\
& \leqq \\
& \leqq \\
& \leqq K\left\|_{p, q}\right\| U^{(k)} \|_{\alpha p^{\prime}, \alpha q^{\prime}}\left(\int_{0}^{t_{1}}\left(\left\|U_{x}^{(k)}\right\|_{\alpha, \alpha}^{\alpha}+\left\|U^{(k)}\right\|_{2, \infty}^{\alpha}\right)\right. \\
& \quad+\|g\|_{p, q}^{\alpha /(\alpha-1)}\left(\int_{0}^{t_{1}}\left(\operatorname{meas} A_{k}(t)\right)^{q^{\prime} / p^{\prime}} d t\right)^{((\alpha-1) / \alpha) \times\left(1 / q^{\prime}\right)} \\
&
\end{aligned}
$$

If we take $t_{1}$ sufficiently so small that

$$
K t_{1}^{\nu}\left(\left\|\theta_{1}\right\|_{p, q}+1\right)<a_{1},
$$

then from $(3.10) \sim(3.13)$ we have

$$
\left\|U^{(k)}\right\|_{2, \infty}^{\alpha}+\left\|U_{x}\right\|_{\alpha, \alpha}^{\alpha} \leqq C\left(1+k^{\alpha}\right)\left(\int_{0}^{t_{1}}\left(\operatorname{meas} A_{k}(t)\right)^{q^{\prime} / p^{\prime}} d t\right)^{1 / q^{\prime}}
$$

where $C$ is a positive constant depending only on $\alpha, M, a_{0},\|b\|,\|c\|,\|d\|$, $\|f\|$ and $\|g\|$.

The following analogous inequality is obtained by the same caluculation as above:

$$
\left\|U^{(k)}\right\|_{2, \infty}^{\alpha}+\left\|U_{x}^{(k)}\right\|_{\alpha, \alpha}^{\alpha} \leqq C\left(1+k^{\alpha}\right)\left(\int_{0}^{t_{1}}\left(\operatorname{meas} B_{k}(t)\right)^{q^{\prime} / p^{\prime}} d t\right)^{1 / q^{\prime}}
$$

for $k \leqq-M_{0}$.

The inequalities (3.14) and (3.15) are used to prove boundedness of weak solutions $u$ (see $\S 4$ ).

In the following, we derive other inequalities for weak solutions which will be used in $\S 5$.

Let $u$ be a bounded weak solution of (1.1), (1.2) and put $\|u\|_{\infty, Q}=M_{1}, \quad c(x, t) M_{1}^{\alpha}+f(x, t)=f_{1}(x, t), \quad d(x, t) M_{1}^{\alpha-1}+g(x, t)=g_{1}(x, t)$ and $e(x, t) M_{1}^{\alpha-1}+h(x, t)=h_{1}(x, t)$.

Then from the condition (1.3), we have

$$
\left\{\begin{array}{l}
p \cdot \mathscr{A}(x, t, u, p) \geqq a_{0}|p|^{\alpha}-f_{1}, \\
|B(x, t, u, p)| \leqq b|p|^{\alpha-1}+g_{1}, \\
|\mathscr{A}(x, t, u, p)| \leqq \bar{a}|p|^{\alpha-1}+h_{1} .
\end{array}\right.
$$


We introduce the notation

$$
\begin{gathered}
K(\rho)=\left\{x|| x-x_{0} \mid<\rho, x_{0} \in \Omega\right\}, \quad \Gamma_{\rho}=K(\rho) \cap \partial \Omega, \\
A_{k, \rho}(t)=\{x \in K(\rho) \mid u(x, t) \geqq k\}, \\
B_{k, \rho}(t)=\{x \in K(\rho) \mid u(x, t) \leqq k\},
\end{gathered}
$$

and for $\rho>\rho^{\prime}$

$$
\zeta=\zeta\left(x ; \rho, \rho^{\prime}\right)= \begin{cases}1 & \text { for } x \in K\left(\rho-\rho^{\prime}\right), \\ \frac{\rho-\left|x-x_{0}\right|}{\rho-\rho^{\prime}} & \text { for } x \in K(\rho)-K\left(\rho^{\prime}\right), \\ 0 & \text { outside } K(\rho),\end{cases}
$$

where $K\left(\rho^{\prime}\right)$ is a concentric cube with $K(\rho)$.

If we put $\Phi(x, t)=u^{(k) \zeta^{\alpha}}$ for $k \geqq \max _{\Gamma \rho \times\left[t_{0}, t_{1}\right]} u$, then

$\Phi \in L^{\infty}\left[0, T ; L^{2}(\Omega)\right] \cap L^{\alpha}\left[0, T ; H_{0}^{1, \alpha}(\Omega)\right]$. (When $K(\rho) \subset \Omega, k$ is an arbitrary number.) Since $u$ is a weak solution of (1.1), (1.2), the equality (1.7) is valid for $\Phi=u^{(k)} \zeta^{\alpha}$, that is for any $t_{0}, t_{1}\left(0 \leqq t_{0}<t_{1} \leqq T\right)$,

$$
\int_{t_{0}}^{t_{1}} \int_{A_{k}, \rho(t)}\left\{u_{t} u^{(k)} \zeta^{\alpha}+\left(u_{x}^{(k)} \zeta^{\alpha}+\alpha \zeta^{\alpha-1} \zeta_{x} u^{(k)}\right) \cdot \mathscr{A}+u^{(k)} \zeta^{\alpha} B\right\} d x d t=0 .
$$

Since $\zeta^{\alpha}$ is independent of the variable $t$, it follows that

$$
u_{t} u^{(k)} \zeta^{\alpha}=\frac{1}{2}\left\{\left(u^{(k)}\right)^{2}\right\}_{t} \quad \text { in } A_{k, \rho}(t)
$$

From the condition (3.16), we see

$$
\begin{aligned}
& u_{x}^{(k)} \zeta^{\alpha} \cdot \mathscr{A} \geqq a_{0}\left|u_{x}^{(k)}\right|^{\alpha} \zeta^{\alpha}-f_{1}, \\
& \alpha u^{(k)} \zeta^{\alpha-1} \zeta_{x} \cdot \mathscr{A} \leqq \alpha \bar{a}\left|u_{x}^{(k)}\right|^{\alpha-1}\left|u^{(k)}\right| \zeta^{\alpha-1}\left|\zeta_{x}\right|+d\left|u^{(k)}\right|^{\alpha-1} \zeta^{\alpha-1}\left|\zeta_{x}\right| h_{1} \\
& \leqq \varepsilon\left|u_{x}^{(k)}\right|^{\alpha} \zeta^{\alpha}+C_{0}\left|u^{(k)}\right|^{\alpha}\left|\zeta_{x}\right|^{\alpha} \\
& +C_{1}\left(\left|u^{(k)}\right|^{\alpha}\left|\zeta_{x}\right|^{\alpha}+h_{1}^{\left.\alpha /(\alpha-1) \zeta^{\alpha}\right)}\right. \text {, }
\end{aligned}
$$

and

$$
\begin{aligned}
u^{(k)} \zeta^{\alpha} B & \leqq b\left|u_{x}^{(k)}\right|^{\alpha-1} \zeta^{\alpha}\left|u^{(k)}\right|+g_{1}\left|u^{(k)}\right| \zeta^{\alpha} \\
& \leqq \varepsilon\left|u_{x}^{(k)}\right|^{\alpha} \zeta^{\alpha}+C_{2} b^{\alpha}\left|u^{(k)}\right|^{\alpha} \zeta^{\alpha}+g_{1}\left|u^{(k)}\right| \zeta^{\alpha}
\end{aligned}
$$

for an arbitrary positive number $\varepsilon$, where $C_{0}, C_{1}$ and $C_{2}$ are constants depending only on $\alpha$ and $\varepsilon$.

Taking $\varepsilon=\frac{a_{0}}{4}$, we have from $(3.17) \sim(3.21)$, 
(3.22) $\frac{1}{2} \int_{A_{k, \rho}(t)}\left(u^{(k)}\right)^{2} \zeta^{\alpha} d x-\frac{1}{2} \int_{A_{k, \rho}\left(t_{0}\right)}\left(u^{(k)}\left(x, t_{0}\right)\right)^{2} \zeta^{\alpha} d x$

$$
\begin{array}{r}
+\frac{a_{0}}{2} \int_{t_{0}}^{t} \int_{A_{k, \rho}(t)}\left|u_{x}\right|^{\alpha} \zeta^{\alpha} d x d t \\
\leqq C_{3}\left\{\int _ { t _ { 0 } } ^ { t } \int _ { A _ { k , \rho } ( t ) } \left\{b^{\alpha}\left|u^{(k)}\right|^{\alpha} \zeta^{\alpha}+g_{1}\left|u^{(k)}\right| \zeta^{\alpha}+\left(f_{1}+h_{1}^{\alpha /(\alpha-1)}\right) \zeta^{\alpha}\right.\right. \\
\left.\left.+\left|u^{(k)}\right|^{\alpha}\left|\zeta_{x}\right|^{\alpha}\right\} d x d t\right\}
\end{array}
$$

for any $t\left(0 \leqq t_{0} \leqq t \leqq t_{1} \leqq T\right)$.

First, we see from Lemma 3.3,

$$
\begin{aligned}
\int_{t_{0}}^{t} \int_{A_{k, \rho}(t)} b^{\alpha}\left|u^{(k)}\right|^{\alpha} \zeta^{\alpha} d x d t \leqq\left\|b^{\alpha}\right\|_{p, q}\left\{\left(t-t_{0}\right)^{\nu \alpha /(\alpha-1)}\left\|u^{(k)} \zeta\right\|_{2, \infty}^{\alpha}\right. \\
+\varepsilon\left\|\left(u^{(k)} \zeta\right)_{x}\right\|_{\alpha, \alpha}^{\alpha}
\end{aligned}
$$

where $\left\|u^{(k)} \zeta\right\|_{2, \infty}^{\alpha}=\max _{t_{0} \leqq t \leqq t_{1}}\left(\int\left(u^{(k)}\right)^{2} d x\right)^{\alpha / 2}$.

Similarly we obtain

$$
\begin{aligned}
& \int_{t_{0}}^{t} \int_{A_{k, \rho}(t)} g_{1}\left|u^{(k)}\right| \zeta^{\alpha} d x d t \\
& \leqq\left\|g_{1}\right\|_{p, q}\left\|u^{(k)} \zeta\right\|_{\alpha p^{\prime}, \alpha q^{\prime}}\left(\int_{t_{0}}^{t}\left(\operatorname{meas} A_{k, \rho}(t)\right)^{q^{\prime} / p^{\prime}} d t\right)^{(\alpha-1) / \alpha q^{\prime}} \\
& \leqq \varepsilon\left(\left\|u_{x}^{(k)} \zeta\right\|_{\alpha, \alpha}^{\alpha}+\left\|u^{(k)} \zeta_{x}\right\|_{\alpha, \alpha}^{\alpha}\right)+C_{4}\left(t-t_{0}\right)^{\nu \alpha /(\alpha-1)} \| u^{(k) \zeta \|_{2, \infty}^{\alpha}} \\
& \quad+C_{5}\left\|g_{1}\right\|_{p, q}^{\alpha /(\alpha-1)}\left(\int_{t_{0}}^{t}\left(\operatorname{meas} A_{k, \rho}(t)\right)^{q^{\prime} / p^{\prime}} d t\right)^{1 / q^{\prime}}
\end{aligned}
$$

and

(3.25)

$$
\begin{aligned}
& \int_{t_{0}}^{t} \int_{A_{k, \rho}(t)}\left(f_{1}+h_{1}^{\alpha /(\alpha-1)}\right) \zeta^{\alpha} d x d t \\
& \quad \leqq\left\|f_{1}+h_{1}^{\alpha /(\alpha-1)}\right\|_{p, q}\left(\int_{t_{0}}^{t}\left(\operatorname{meas} A_{k, \rho}(t)\right)^{q^{\prime} / p^{\prime}} d t\right)^{1 / q^{\prime}} .
\end{aligned}
$$

From $(3.22) \sim(3.25)$, by putting $\varepsilon=\frac{a_{0}}{4\left(1+\|b\|_{p, q}\right)}$, it holds

(3.26) $\frac{1}{2} \int_{A_{k, \rho}(t)}\left(u^{(k)}\right)^{2} \zeta^{\alpha} d x-\frac{1}{2} \int_{A_{k, \rho}\left(t_{0}\right)}\left(u^{(k)}\left(x, t_{0}\right)\right)^{2} \zeta^{\alpha} d x$

$$
\begin{aligned}
& +\frac{a_{0}}{4} \int_{t_{0}}^{t} \int_{A_{k, \rho}(t)}\left|u_{x}^{(k)}\right|^{\alpha} \zeta^{\alpha} d x d t \\
\leqq & \mathscr{C}\left\{\int_{t_{0}}^{t} \int_{A_{k, \rho}(t)}\left|u^{(k)}\right|^{\alpha}\left|\zeta_{x}\right|^{\alpha} d x d t+\max _{t_{0} \leqq t \leqq t_{1}}\left(\int_{A_{k, \rho}(t)}\left|u^{(k)}\right|^{2} \zeta^{2} d x\right)^{\alpha / 2}\right.
\end{aligned}
$$




$$
\left.\times\left(t-t_{0}\right)^{\nu \alpha /(\alpha-1)}+\left(\int_{t_{0}}^{t}\left(\operatorname{meas} A_{k, \rho}(t)\right)^{q^{\prime} / p^{\prime}} d t\right)^{1 / q^{\prime}}\right\}=I(t)
$$

for any $t\left(t_{0} \leqq t \leqq t_{1}\right)$. From this we have the following two inequalities

$$
\max _{t_{0} \leqq t \leqq t_{1}} \int_{A_{k, \rho}(t)}\left(u^{(k)}(x, t)\right)^{2} \zeta^{\alpha} d x \leqq I\left(t_{1}\right)+\int_{A_{k, \rho}\left(t_{0}\right)}\left(u^{(k)}\left(x, t_{0}\right)\right)^{2} \zeta^{\alpha} d x,
$$

$$
\int_{t_{0} A_{k, \rho}(t)}^{t_{1}}\left|u_{x}^{(k)}\right|^{\alpha} \zeta^{\alpha} d x d t \leqq I\left(t_{1}\right)
$$

for any $t_{1}\left(0 \leqq t_{0}<t_{1} \leqq T\right)$.

\section{§ 4. Boundedness of weak solutions.}

In this section we concern with boundedness of a weak solution $u$ when $u$ is bounded on the parabolic boundary $\partial Q=\partial \Omega \times(0, T] \cup \Omega \times$ $\{t=0\}$, that is, when $\psi(x, t)$ and $\phi(x)$ are bounded.

LEMMA 4.1 (Stampacchia [5]). Let $\Xi(k)$ be a non-negative and nonincreasing function defined for $k \geqq k_{0}$. If the inequality

$$
\boldsymbol{g}(h) \leqq \frac{C}{(h-k)^{s}}[\boldsymbol{g}(k)]^{\beta}
$$

holds for $h>k \geqq k_{0}$ and $\beta>1$, then

$$
\Xi\left(k_{0}+d^{s}\right)=0 \text {, }
$$

where $d^{s}=C\left[\Xi\left(k_{0}\right)\right]^{\beta-1} 2^{s \beta /(\beta-1)}$.

Now we can prove the following.

THEOREM 4.1. Suppose that $\psi(x, t)$ and $\phi(x)$ are bounded. Then $a$ weak solution of the problem (1.1), (1.2) is bounded in $Q$.

Proof. Let $M_{0}$ be a positive constant such that

$$
|\psi(x, t)| \leqq M_{0} \quad \text { and } \quad|\phi(x)| \leqq M_{0} \quad\left(M_{0}>1\right)
$$

and let

$$
U=\frac{u}{M}, \quad \text { where } M=\max _{0 \leqq t \leqq T}\left(\int_{\Omega} u^{2} d x\right)^{1 / 2}
$$

Then the inequality (3.14) and (3.15) hold for $U$.

Now, put $k_{h}=M_{0}\left(2-\frac{1}{2^{h}}\right) \quad(h=0,1,2, \cdots)$ and 


$$
\mu(k)=\int_{t_{0}}^{t_{1}}\left(\operatorname{meas} A_{k}(t)\right)^{q^{\prime} / p^{\prime}} d t .
$$

Then it follows that

$$
\begin{aligned}
& \left(k_{h+1}-k_{h}\right)^{\alpha} \mu\left(k_{h+1}\right)^{\alpha / q^{\prime} \kappa} \leqq\left(\int_{0}^{t_{1}}\left(\int_{A_{k_{h}}(t)}\left(u_{h}^{\left(k_{h}\right)}\right)^{\alpha \kappa p^{\prime}} d x\right)^{q^{\prime} / p^{\prime}} d t\right)^{\alpha / \alpha \kappa q^{\prime}} \\
& =\left\|u^{\left(k_{h}\right)}\right\|_{\alpha \kappa p^{\prime}, \alpha \kappa q^{\prime}}^{\alpha} \leqq K t^{\nu}\left(\left\|u^{\left(k_{h}\right)}\right\|_{2, \infty}^{\alpha}+\left\|u_{x}^{\left(k_{h}\right)}\right\|_{\alpha, \alpha}^{\alpha}\right) \leqq C k_{h}^{\alpha} \mu\left(k_{h}\right)^{\alpha / q^{\prime}},
\end{aligned}
$$

where $C$ is a positive constant depending only on $\alpha, M_{0}, M, a_{0},\|b\|,\|c\|$, $\|d\|,\|f\|$ and $\|g\|$.

If we put $\Xi(k)=\mu(k)^{\alpha / q^{\prime} k}$, then

$$
\left(k_{h+1}-k_{h}\right) \Xi\left(k_{h+1}\right) \leqq C k_{h}\left[\Xi\left(k_{h}\right)\right]^{\kappa} .
$$

Since $\kappa>1$, from the preceding lemma 4.1 we have

$$
\Xi\left(k_{0}+d^{s}\right)=0
$$

that is, $u(x, t)$ is bounded from above in $\Omega \times\left(0, t_{1}\right]$.

Similarly, from the inequality (3.15) we see that $u(x, t)$ is bounded from below in $\Omega \times\left(0, t_{1}\right]$.

Repeating the same argument on $\Omega \times\left(N t_{1},(N+1) t_{1}\right]$ inductively, we conclude that $u$ is bounded in $Q$.

\section{§5. Hölder continuity of weak solutions.}

In this section we prove Hölder continuity of a weak solution $u$ of the problem (1.1), (1.2). The method presented here is based on the idea of [3].

Throughout this section, we assume that there is a positive constant $M_{1}$ such that $|u| \leqq M_{1}$ in $Q$.

First we shall state some lemmas.

LEMma 5.1 (Theorem 6.3 in [5]). Let $u(x) \in H^{1,2}(K(\rho))$ and let $A(k, \rho)$ $=\{x \in K(\rho) \mid u(x) \geqq k\}$. If there exist two constants $k_{0}$ and $\theta$ with $0 \leqq \theta<1$ such that meas $A\left(k_{0}, \rho\right)<\theta$ meas $K(\rho)$, then the following inequality holds:

$$
(h-k)[\text { meas } A(h, \rho)]^{1-1 / n} C \int_{[A(k, \rho)-A(h, \rho)]}\left|u_{x}(t)\right| d t
$$

for $h>k>k_{0}$, where $C$ is a positive constant depending only on $\theta$ and $n$. 
LEMMA 5.2. Suppose that meas $A_{k, \rho}\left(t_{0}\right) \leqq \frac{1}{2} \kappa_{n} \rho^{n}$, where $\kappa_{n}=$ meas $K(1)$. Then for any $\beta$ in $\left(\frac{1}{\sqrt{2}}, 1\right)$, there exist positive numbers $a$ and $\theta(0 \leqq \theta<1)$ depending only on $\beta$ such that if

$$
k \geqq \max _{\substack{x \in \partial \Omega \cap K(\rho) \\ t \in\left[t_{0}, t_{0}+a_{\rho} \alpha\right]}} u(x, t) \text { and } 2 M_{1} \geqq H=\max _{\substack{x \in A_{k}, \rho^{\prime}(t) \\ t \in\left[t_{0}, t_{0}+a_{\rho} \alpha\right]}}(u(x, t)-k)>\rho^{r},
$$

where $\gamma=1-\left(\frac{n}{\alpha p}+\frac{\alpha n-2 n+2 \alpha}{2 \alpha q}\right)$ when $\alpha<n$ and $\gamma=1-\left(\frac{1}{p}+\right.$ $\left.\frac{\alpha}{2 q}\right)$ when $\alpha \geqq n$, then

$$
\operatorname{meas} A_{k+\beta H, \rho}(t)<\theta \text { meas } K(\rho)
$$

for $t \in\left[t_{0}, t_{0}+a \rho^{\alpha}\right]$.

Proof. We choose $\zeta(x)$ as follows:

$$
\zeta(x ; \rho, \rho-\sigma \rho)= \begin{cases}1 & \text { for } x \in K(\rho-\sigma \rho), \\ \frac{\rho-\left|x-x_{0}\right|}{\sigma \rho} & \text { for } x \in K(\rho)-K(\rho-\sigma \rho), \\ 0 & \text { outside of } K(\rho),\end{cases}
$$

where $\sigma$ is any number in the interval $(0,1)$. For such a $\zeta$ and $t \epsilon$ $\left[t_{0}, t_{0}+a \rho^{\alpha}\right]$, it follows from the inequality (3.27) that

$(\beta H)^{2}\left(\operatorname{meas} A_{k+\beta H, \rho-\sigma \rho}(t)\right)$

$$
\begin{aligned}
& \leqq \int_{A_{k, \rho-\sigma \rho}(t)}(u-k)^{2} d x \leqq \int_{A_{k, \rho}(t)}\left(u^{(k)}\right)^{2} \zeta^{\alpha} d x \\
& \leqq \mathscr{C}\left\{\int_{t_{0}}^{t} \int_{A_{k, \rho}(t)}\left|u^{(k)}\right|^{\alpha}\left|\zeta_{x}\right|^{\alpha} d x d t+\max _{t}\left(\int_{A_{k, \rho}(t)}\left|u^{(k)}\right|^{2} \zeta^{2} d x\right)^{\alpha / 2}\left(t-t_{0}\right)^{\alpha \nu /(\alpha-1)}\right. \\
& \left.+\left(\int_{t_{0}}^{t}\left(\operatorname{meas} A_{k, \rho}(t)\right)^{q^{\prime} / p^{\prime}} d t\right)^{1 / q^{\prime}}\right\}+\int_{A_{k, \rho}\left(t_{0}\right)}\left(u^{(k)}\left(x, t_{0}\right)\right)^{2} \zeta^{\alpha} d x .
\end{aligned}
$$

Since, from the hypotheses,

$$
\begin{aligned}
& \int_{t_{0}}^{t} \int_{A_{k, \rho}(t)}\left(u^{(k)}\right)^{\alpha}\left|\zeta_{x}\right|^{\alpha} d x \leqq \frac{H^{\alpha}}{(\sigma \rho)^{\alpha}}\left(t-t_{0}\right) \kappa_{n} \rho^{n}, \\
& \left(t-t_{0}\right)^{\alpha \nu /(\alpha-1)}\left\|u^{(k) \zeta}\right\|_{2, \infty}^{\alpha} \leqq H^{\alpha}\left(t-t_{0}\right)^{\alpha \nu /(\alpha-1)} \kappa_{n}^{\alpha / 2} \rho^{\alpha n / 2} \leqq H^{\alpha} \kappa_{n}^{\alpha / 2} \rho^{n} a^{\alpha \nu /(\alpha-1)}, \\
& \left(\int_{t_{0}}^{t}\left(\operatorname{meas} A_{k, \rho}(t)\right)^{q^{\prime} / p^{\prime}} d t\right)^{1 / q^{\prime}} \leqq\left(t-t_{0}\right)^{1 / q^{\prime}}\left(\kappa_{n} \rho^{n}\right)^{1 / p^{\prime}} \\
& \leqq\left(t-t_{0}\right)^{1 / q^{\prime}} H^{\alpha} \kappa_{n}^{1 / p^{\prime}} \rho^{n / p^{\prime}-\alpha \gamma} \leqq a^{1 / q^{\prime}} H^{\alpha} \kappa_{n}^{1 / p^{\prime}} \rho^{n}
\end{aligned}
$$


and

$$
\int_{A_{k, \rho}\left(t_{0}\right)}\left\{u^{(k)}\left(x, t_{0}\right)\right\}^{2} \zeta^{\alpha} d x \leqq \frac{1}{2} \kappa_{n} \rho^{n} H^{2},
$$

it follows that

$$
\begin{aligned}
& \operatorname{meas} A_{k+\beta H, \rho-\sigma \rho}(t) \\
& \qquad \leqq \frac{\mathscr{C}}{\beta^{2}} H^{\alpha-2}\left\{\frac{a}{\sigma^{\alpha}}+a^{1 / q^{\prime}} \kappa_{n}^{1 / p^{\prime}-1}+a^{\alpha \nu /(\alpha-1)} \kappa_{n}^{-1}\right\} \kappa_{n} \rho^{n}+\frac{1}{2 \beta^{2}} \kappa_{n} \rho^{n} .
\end{aligned}
$$

Now we take $\beta \in\left(\frac{1}{\sqrt{2}}, 1\right)$ and choose $\theta(0 \leqq \theta<1)$ and $\sigma>0$ such that the inequality

$$
\frac{1}{2 \beta^{2}}<\theta(1-\sigma)^{n}
$$

holds. Then if we choose the number $a$ sufficiently small, the right hand side of (5.2) is smaller than $\theta \kappa_{n}(1-\sigma)^{n} \rho^{n}$. Hence we obtain

$$
\text { meas } A_{k+\beta H, \rho-\sigma \rho}(t) \leqq \theta \text { meas } K((1-\sigma) \rho) \quad \text { for } t \in\left[t_{0}, t_{0}+a \rho^{\alpha}\right],
$$

from which we have the lemma.

In what follows, we take $\beta=\frac{3}{4}$.

We introduce standard cylinders $Q(r \rho)$ whose bases are the ball $K(r \rho)$ with heights equal to $a(r \rho)^{\alpha}$, where $a$ is a positive constant chosen in Lemma 5.2, that is,

$$
Q(r \rho)=K(r \rho) \times\left[t_{1}-a(r \rho)^{\alpha}, t_{1}\right], \quad t_{1}>a(r \rho)^{\alpha} .
$$

Write

$$
\mu_{1}=\max _{Q(8 \rho)} u, \quad \mu_{2}=\min _{Q(8 \rho)} u \quad \text { and } \quad \omega=\mu_{1}-\mu_{2} .
$$

LEMMA 5.3. For any $\theta_{1}>0$ and for any $\rho<1$, there exists an $s\left(\theta_{1}\right)>0$ such that for any cylinder $Q(8 \rho) \subset Q$, either

$$
\omega<2^{s} \rho^{r}
$$

where $\gamma=1-\left(\frac{n}{\alpha p}+\frac{\alpha n-2 n+2 \alpha}{2 \alpha q}\right)$ when $n>\alpha$, and $\gamma=1-\left(\frac{1}{p}+\frac{\alpha}{2 q}\right)$ when $\alpha \geqq n$, or 


$$
\int_{t_{1}-a(4 \rho) \alpha}^{t_{1}} \operatorname{meas} A_{\mu_{1}-\left(\omega / 2^{s+1), 4 \rho}\right.}(t) d t \leqq \theta_{1} \rho^{n+\alpha},
$$

or

$$
\int_{t_{1}-a(4 \rho) \alpha}^{t_{1}} \operatorname{meas} B_{\mu_{2}+\left(\omega / 2^{s+1), 4 \rho}\right.}(t) d t \leqq \theta_{1} \rho^{n+\alpha}
$$

Proof. Let $r$ be an integer $>2$. Since $\mu_{2}+\frac{\omega}{2^{r}}<\mu_{1}-\frac{\omega}{2^{r}}$, it is obvious that at least one of the following inequalities holds:

$$
\operatorname{meas} A_{\mu_{1}-(\omega / 2 r), 4 \rho}\left(t_{1}-a(4 \rho)^{\alpha}\right) \leqq \frac{1}{2} \kappa_{n}(4 \rho)^{n}
$$

and

$$
\text { meas } B_{\mu_{2}+\left(\omega / 2^{r}\right), 4 \rho}\left(t_{1}-a(4 \rho)^{\alpha}\right) \leqq \frac{1}{2} \kappa_{n}(4 \rho)^{n}
$$

Suppose for example that the first one holds. We shall prove that then (5.5) will be satisfied if $\omega>2^{s} \rho^{\gamma}$.

From Lemma 5.2, for all $t \in\left[t_{1}-a(4 \rho)^{\alpha}, t_{1}\right]$

$$
\operatorname{meas} A_{\mu_{1}-(\omega / 2 r+2), 4 \rho}(t)<\theta \kappa_{n}(4 \rho)^{n} \text {, }
$$

so that, for such a $t$, Lemma 5.1 may be applied on account of the fact that

$$
h>k \geqq u_{1}-\frac{\omega}{2^{r+2}} .
$$

We denote by $D_{\lambda_{\ell}}(t)$ the set

$$
A_{\mu_{1}-\{\omega / 2 \ell\}, 4 \rho}(t)-A_{\mu_{1}-\{\omega / 2 \ell+1\}, 4 \rho}(t), \quad r+2 \leqq \ell \leqq s .
$$

Using Lemma 5.1, we have

$$
\begin{aligned}
\frac{\omega}{\kappa_{n}^{1 / n}(4 \rho) 2^{\ell+1}} \operatorname{meas} A_{\mu_{1}-(\omega / 2 \ell+1), 4 \rho}(t) & \leqq \frac{\omega}{2^{\rho+1}}\left[\operatorname{meas} A_{\mu_{1}-(\omega / 2 \ell+1), 4 \rho}(t)\right]^{1-1 / n} \\
& \leqq \mathscr{C}\left[_{D_{\lambda \ell}(t)}\left|u_{x}\right| d x\right.
\end{aligned}
$$

From this we have, putting $t_{0}=t_{1}-a(4 \rho)^{\alpha}$,

$$
\frac{\omega^{\alpha}}{2^{\alpha(\ell+3)} \kappa_{n}^{\alpha / n} \rho^{\alpha}}\left\{\int_{t_{0}}^{t_{1}}\left(\operatorname{meas} A_{\mu_{1}-(\omega / 2 \ell+1), 4 \rho}(t)\right) d t\right\}^{\alpha}
$$




$$
\leqq \mathscr{C}^{\alpha}\left(\int_{t_{0}}^{t_{1}} \int_{D_{\lambda \ell}}\left|u_{x}\right|^{\alpha} d x d t\right)\left(\int_{t_{0}}^{t_{1}} \operatorname{meas} D_{\lambda \ell}(t) d t\right)^{\alpha-1}
$$

On the other hand, if we take $\zeta(x)=\zeta(x ; 8 \rho, 4 \rho)$ in (3.28) with $t_{0}=t_{1}-a(4 \rho)^{\alpha}$, then we obtain

$$
\begin{aligned}
& \int_{t_{0}}^{t_{1}} \int_{D_{\lambda \ell}}\left|u_{x}\right|^{\alpha} d x d t \leqq \int_{t_{0}}^{t_{1}} \int_{\left.A_{\mu_{1}-(\omega / 2} \ell\right), 8 \rho(t)}\left|u_{x}\right|^{\alpha} \zeta^{\alpha} d x d t \\
& \leqq \mathscr{C}\left\{a(4 \rho)^{\alpha}\left[\frac{\omega^{\alpha}}{2^{\alpha \ell} \rho^{\alpha}} \kappa_{n}(8 \rho)^{n}\right]+\left(a 4^{\alpha} \rho^{\alpha}\right)^{\alpha \nu /(\alpha-1)} \frac{\omega^{\alpha}}{2^{\alpha \ell}}(8 \rho)^{n \alpha / 2} \kappa_{n}^{n / 2}\right. \\
&\left.\quad+\left(a 4^{\alpha} \rho^{\alpha}\right)^{1 / q^{\prime}}\left(8^{n} \rho^{n} \kappa_{n}\right)^{1 / p^{\prime}}\right\} \\
& \leqq C_{1} \omega^{\alpha}\left\{\rho^{n}+\rho^{\alpha(\alpha \nu / \alpha-1)+\alpha n / 2}+\rho^{\alpha-(\alpha / q)+n-(n / q)-\alpha \gamma}\right\} \leqq C_{1} \omega^{\alpha} \rho^{n},
\end{aligned}
$$

where $C_{1}$ is a positive constant depending only on $a, \kappa_{n}$ and $\mathscr{C}$ in (3.28), and we used the fact that

$$
\alpha\left(\frac{\alpha \nu}{\alpha-1}\right)+\frac{n \alpha}{2} \geqq n, \quad \alpha-\frac{\alpha}{q}+n-\frac{n}{p}-\alpha \gamma \geqq n .
$$

Therefore the inequalities (5.7) and (5.8) yield

$$
\left(\int_{t_{0}}^{t_{1}} \operatorname{meas} A_{\mu_{1}-\left(\omega / 2^{s+1), 4 \rho}\right.}(t) d t\right)^{\alpha / \alpha-1} \leqq C_{2}\left(\rho^{n+\alpha}\right)^{\alpha /(\alpha-1)} \int_{t_{0}}^{t_{1}} \operatorname{meas} D_{\lambda_{\ell}}(t) d t .
$$

We sum up these inequalities with respect to $\ell$ from $r+2$ to $s$ and obtain

$$
\begin{aligned}
& (s-r-1)\left(\int_{t_{0}}^{t_{1}} \operatorname{mean} A_{\mu_{1}-\left(\omega / 2^{s+1), 4}\right.}(t) d t\right)^{\alpha /(\alpha-1)} \\
& \quad \leqq C_{2}\left(\rho^{n+\alpha}\right)^{1 /(\alpha-1)} \int_{t_{0}}^{t_{1}} K(4 \rho) d t=C_{2} 2^{2 n+2 \alpha} a\left(\rho^{n+\alpha}\right)^{1 /(\alpha-1)} \rho^{n+\alpha}=C_{3}\left(\rho^{n+\alpha}\right)^{\alpha /(\alpha-1)} .
\end{aligned}
$$

Hence we have

$$
\int_{t_{0}}^{t_{1}} \operatorname{meas} A_{\mu_{1}-\left(\omega / 2^{s+1), 4 \rho}\right.}(t) d t \leqq\left(\frac{C_{3}}{s-r+1}\right)^{(\alpha-1) / \alpha} \rho^{n+\alpha} .
$$

Therefore we have the inequality (5.5) by choosing $s$ such that

$$
\left(\frac{C_{3}}{s-r+1}\right)^{(\alpha-1) / \alpha}=\theta_{1} \text {. }
$$

LEMma 5.3'. Suppose that the oscillation $\omega_{1}=\operatorname{osc}\{u, Q(8 \rho)\}$ of $u$ on the intersection $\Gamma(8 \rho)$ of the cylinder $Q(8 \rho)$ with $\Gamma$ satisfies $\omega_{1} \leqq L \rho^{\varepsilon}$, for some positive number $\varepsilon$. 
Then for any $\theta_{1}>0$ one can find an $s\left(\theta_{1}\right)>0$ such that for any pair of coaxial cylinders $Q(4 \rho)$ and $Q(8 \rho)$ satisfying the condition

$$
\text { meas }[K(4 \rho)-K(4 \rho) \cap \Omega] \geqq b_{1} \rho^{n},
$$

at least one of the three inequalities $\omega=\operatorname{osc}\{u, Q(8 \rho)\}<2^{s} \rho^{s_{1}}\left(\varepsilon_{1}=\min \gamma, \varepsilon\right)$, (5.5) and (5.6) holds.

The proof is analogous to the proof of Lemma 5.3, so we omit it here.

Lemma 5.4. There exists $a \theta_{2}>0$ such that if

$$
\max _{t \in\left[t_{1}-a(2 \rho) \alpha, t_{1}\right]} \operatorname{meas} A_{k, 2 \rho}(t)<\theta_{2} \rho^{n} \quad \text { in } \quad Q(2 \rho)
$$

and if

$$
k \geqq \max _{\Gamma(2 \rho)} u(x, t), \quad H=\max _{Q(2 \rho)}(u-k)>\rho^{r},
$$

then

$$
\operatorname{meas} A_{k+H / 2, \rho}(t)=0, \quad t \in\left[t_{1}-a \rho^{\alpha}, t_{1}\right] .
$$

Proof. We introduce the notation

$$
\begin{array}{cc}
k_{h}=k+\frac{H}{2}-\frac{H}{2^{h+1}}, \quad t_{h}=t_{1}-a \rho^{\alpha}-\frac{a \rho^{\alpha}}{2^{h}}, & \rho_{h}=\rho+\frac{\rho}{2^{h}}, \\
\mu_{h}=\max _{t \in\left[t_{h}, t_{1}\right]}\left(\operatorname{meas} A_{k_{h}, \rho_{h}}(t)\right), \quad \zeta_{h}=\zeta\left(x ; \rho_{h}, \rho_{h+1}\right), & (h=0,1,2, \cdots)
\end{array}
$$

Evidently, for any $h$.

$$
\begin{aligned}
\left(k_{h+1}-k_{h}\right)^{\alpha} \operatorname{meas} A_{k_{h+1}, \rho_{h+1}}(t) & \leqq \int_{A_{k_{h}, \rho_{h+1}(t)}}\left(u-k_{h}\right)^{\alpha} d x \\
& \leqq \int_{A_{k_{h}, \rho_{h}(t)}}\left(u^{\left(k_{h}\right)}\right)^{\alpha} \zeta_{h}^{\alpha} d x .
\end{aligned}
$$

Integrating by $t$ and using Lemma 2.4 and (3.28) we have

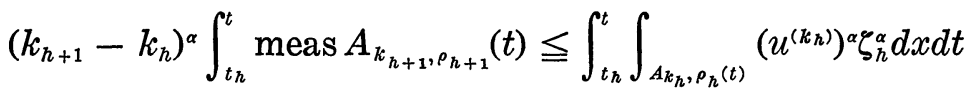

$$
\begin{aligned}
& \leqq K\left(\int_{t_{n}}^{t} \int_{A_{k_{h}}, \rho_{h}(t)}\left(\left|u_{x}^{\left(k_{n}\right)}\right|^{\alpha} \zeta_{h}^{\alpha}+\left|u^{\left(k_{n}\right)}\right|^{\alpha}\left|\left(\zeta_{h}\right)_{x}\right|^{\alpha}\right) d x d t\right) \mu_{h}^{\alpha / n} \\
& \leqq C_{1}\left\{\frac{t-t_{h}}{\left(\rho_{h}-\rho_{h+1}\right)^{\alpha}} H^{\alpha} \mu_{h}+H^{\alpha} \mu_{h}^{\alpha / 2}+H^{\alpha} \frac{\left(t-t_{h}\right)^{1 / q^{\prime}} \mu_{h}^{1 / p^{\prime}}}{\rho^{\alpha \tau}}\right\} \mu_{h}^{\alpha / n}
\end{aligned}
$$

for any $t>t_{h}$. Choose $t=t_{h+1}$. Then we obtain 


$$
\mu_{h+1} \leqq \frac{C_{1}}{\left(k_{h+1}-k_{h}\right)^{\alpha}}\left\{\frac{\mu_{h}^{1+\alpha / n}}{\left(t_{h+1}-t_{h}\right)}+\frac{\mu_{h}^{\alpha / 2+\alpha / n}}{\left(t_{h+1}-t_{h}\right)}+\frac{\mu_{h}^{1+\alpha / n-1 / p}}{\rho^{\alpha \gamma}\left(t_{h+1}-t_{h}\right)^{1 / q}}\right\},
$$

from which, taking account of the definition of $k_{h}, \rho_{h}, t_{h}$ we arrive at the inequality

$$
y_{h+1} \leqq C_{2} 2^{\alpha h} y_{h}^{1+s}
$$

where $\varepsilon=\frac{\alpha}{n}-\frac{1}{p}>0, y_{h}=\frac{\mu_{h}}{\rho^{n}}$ and $C_{2}$ is a positive constant depending only on $\mathscr{C}$ in (3.28).

Now we choose $\theta_{2}$ such as

$$
\theta_{2} \leqq \frac{1}{C_{2} 2^{2 \alpha / s}}
$$

Then if $y_{0} \leqq \theta_{2}$, we have

$$
y_{h} \leqq \theta_{2} 2^{-\alpha h / \varepsilon} .
$$

Taking such a $\theta_{2}$ and letting $h$ tend to $+\infty$, we have that $y_{h} \rightarrow 0$, i.e., that

$$
\text { meas } A_{k+H / 2, \rho}(t)=0 \quad \text { for } \quad t \in\left[t_{1}-a \rho^{\alpha}, t_{1}\right] .
$$

In what follows we fix $\theta_{2}\left(1>\theta_{2}>0\right)$ satisfying condition (5.11) and a sufficiently small number $\rho_{0}$ such that

$$
\mathscr{C}\left(2 M_{1}\right)^{\alpha-2} a^{\alpha \nu /(\alpha-1)}\left(4 \rho_{0}\right)^{\alpha^{2} / /(\alpha-1)+(n \alpha / 2)} \rho_{0}^{-n}=\frac{\theta_{2}}{2},
$$

where $\mathscr{C}$ is a positive constant in (3.27) of (3.28).

LEMMA 5.5. For $\theta_{2}>0$, there exists $a \theta_{1}>0$ such that if

$$
k>\max _{\Gamma(4 \rho)} u(x, t), \quad H=\max _{Q(4 \rho)}(u-k)>\rho^{r}, \quad \rho \leqq \rho_{0},
$$

then inequality

$$
\int_{t_{1}-a(4 \rho) \alpha}^{t_{1}} \operatorname{meas} A_{k, 4 \rho}(t) d t<\theta_{1} \rho^{n+\alpha}
$$

implies

$$
\text { meas } A_{k+H / 2,2 \rho}(t) \leqq \theta_{2} \rho^{n}, \quad t \in\left[t_{1}-a(2 \rho)^{\alpha}, t_{1}\right] .
$$

Proof. Put $\zeta=\zeta(x ; 4 \rho, 2 \rho)$. Then we have from (3.27) 
$\left(\frac{H}{2}\right)^{2}$ meas $A_{k+H / 2, \rho}(t) \leqq \mathscr{C}\left\{\frac{H^{\alpha}}{\rho^{\alpha}} \int_{\tau}^{t} \operatorname{meas} A_{k, 4 \rho}(t) d t\right.$

$$
\begin{aligned}
& +(t-\tau)^{\alpha \nu /(\alpha-1)} H^{\alpha}\left(\max _{t} \operatorname{meas} A_{k, 4 \rho}(t)\right)^{\alpha / 2} \\
& \left.+\left(\int_{\tau}^{t}\left(\operatorname{meas} A_{k, 4 \rho}(t)\right)^{q^{\prime} / p^{\prime}} d t\right)^{1 / q^{\prime}}\right\} \\
& +H^{2} \text { meas } A_{k, 4 \rho}(\tau), \quad t_{1}-a(4 \rho)^{\alpha} \leqq \tau \leqq t \leqq t_{1} .
\end{aligned}
$$

From (5.12), it is clear that

$$
\frac{1}{\rho^{\alpha}} \int_{z}^{t} \operatorname{meas} A_{k, 4 \rho}(t) d t \leqq \theta_{1} \rho^{n} .
$$

Since $t-\tau<a(4 \rho)^{\alpha}$ and $\rho \leqq \rho_{0}$, it holds that

$$
\begin{aligned}
& (t-\tau)^{\alpha \nu /(\alpha-1)}\left(\max _{t} \operatorname{meas} A_{k, 4 \rho}(t)\right)^{\alpha / 2} \\
& \quad \leqq a^{\alpha \nu /(\alpha-1)}(4 \rho)^{\alpha \nu /(\alpha-1)+\alpha n / 2} \leqq \frac{1}{2} \theta_{2} \rho^{n}\left(\mathscr{C}(2 M)^{\alpha-2}\right)^{-1} .
\end{aligned}
$$

If $q^{\prime} \geqq p^{\prime}$, then

$$
\begin{aligned}
\left(\int_{\tau}^{t}\left(\operatorname{meas} A_{k, 4 \rho}(t)\right)^{q^{\prime} / p^{\prime}} d t\right)^{1 / q^{\prime}} & \leqq\left(\int_{\tau}^{t} \operatorname{meas} A_{k, 4 \rho}(t) d t\right)^{1 / q^{\prime}}(4 \rho)^{n / p^{\prime}-n / q^{\prime}} \\
& \leqq 4^{n / p^{\prime}-n / q^{\prime}} \theta_{1}^{1 / q^{\prime}} \rho^{n+\alpha \gamma^{\prime}}
\end{aligned}
$$

where $\gamma^{\prime}=1-\left(\frac{n}{\alpha p}+\frac{1}{q}\right)$.

On the other hand, if $p^{\prime}>q^{\prime}$, then the Hölder's inequality yields

$$
\begin{aligned}
\left(\int_{\tau}^{t}\left(\operatorname{meas} A_{k, 4 \rho}(t)\right)^{q^{\prime} / p^{\prime}} d t\right)^{1 / q^{\prime}} & \leqq\left(\int_{\tau}^{t}\left(\operatorname{meas} A_{k, 4 \rho}(t)\right) d t^{1 / p^{\prime}}(t-\tau)^{1 / q^{\prime}-1 / p^{\prime}}\right. \\
& \leqq\left\{a(4 \rho)^{\alpha}\right\}^{1 / q^{\prime}-1 / p^{\prime}} \theta_{1}^{1 / p^{\prime}} \rho^{(n+\alpha) / p^{\prime}} \leqq\left(4^{\alpha}\right)^{1 / q^{\prime}-1 / p^{\prime}} \theta_{1}^{1 / p^{\prime}} \rho^{n+\alpha \gamma^{\prime}}
\end{aligned}
$$

Thus, putting $\theta_{1}^{r}=\max \left(\theta_{1}^{1 / p^{\prime}}, \theta_{1}^{1 / q^{\prime}}\right)$ and $C_{1}=\max \left(4^{\alpha\left(1 / q^{\prime}-1 / p^{\prime}\right)}, 4^{n\left(1 / p^{\prime}-1 / q^{\prime}\right)}\right)$, we obtain

$$
\left(\int_{\tau}^{t}\left(\operatorname{meas} A_{k, 4 \rho}(t)\right)^{q^{\prime} / p^{\prime}} d t\right)^{1 / q^{\prime}} \leqq C_{1} \theta_{1}^{r} \rho^{n+\alpha \gamma^{\prime}}
$$

Finally we choose $\tau$ in the interval $\left[t_{1}-a(4 \rho)^{\alpha}, t_{1}-a(2 \rho)^{\alpha}\right]$ such that

$$
\operatorname{meas} A_{k, 4 \rho}(\tau) \leqq \frac{\theta_{1} \rho^{n}}{\left(4^{\alpha}-2^{\alpha}\right) a}
$$

Then, from $(5.13) \sim(5.18)$ we have 


$$
\text { meas } A_{k+H / 2,2 \rho}(t) \leqq \mathscr{C}(2 M)^{\alpha-2}\left[\theta_{1}+C_{1} \theta_{1}^{r}+\frac{\theta_{1}}{\left(4^{\alpha}-2^{\alpha}\right) a}+\frac{\theta_{2}}{2(2 M)^{\alpha-2}}\right] \rho^{n}
$$

From (5.19), we obtain the lemma, while $\theta_{1}$ satisfies

$$
\mathscr{C}(2 M)^{\alpha-1}\left[\theta_{1}+C_{1} \theta_{1}^{r}+\frac{\theta_{1}}{\left(4^{\alpha}-2^{\alpha}\right) a}\right] \leqq \frac{1}{2} \theta_{2} .
$$

We put $\mu_{1}(\rho)=\max _{Q(\rho)} u, \mu_{2}(\rho)=\min _{Q(\rho)} u$ and $\omega(\rho)=\mu_{1}(\rho)-\mu_{2}(\rho)$. Then the following Lemma was proved by G. Stampacchia [5]:

LEMMA 5.6. If $\omega(\rho) \leqq \eta \omega(8 \rho)$ with $0<\eta<1$, then there exist $a$ constant $\lambda$ in interval $(0,1)$ and positive constant $K$ such that

$$
\omega(\rho) \leqq K \rho^{\lambda} \text {. }
$$

Now we can prove the main theorem:

THEOREM 5.1. A weak solution $u$ of the problem (1.1), (1.2) is Hölder continuous in $Q$.

Proof. Let $\left(x_{0}, t_{1}\right)$ be any point of $Q$ and choose $\rho_{0}>0$ so small that $Q\left(8 \rho_{0}\right)$ is contained in $Q$, where $Q\left(8 \rho_{0}\right)=K\left(8 \rho_{0}\right) \times\left(t_{1}-a\left(8 \rho_{0}\right)^{\alpha}, t_{1}\right]$ and $K\left(8 \rho_{0}\right)=\left\{x \in \Omega|| x-x_{0} \mid<8 \rho_{0}\right\}$.

First we choose $\theta_{2}$ as in Lemma 5.4 and we choose $\theta_{1}$ as in Lemma 5.5. Then we take $s\left(\theta_{1}\right)$ as in Lemma 5.3.

Now suppose that $\omega(8 \rho) \geqq 2^{s+2} \rho^{\gamma}$. Then either the inequality (5.5) or (5.6) in Lemma 5.3 holds. If the inequality (5.5) is valid, then from Lemma 5.5, we have

$$
\text { meas } A_{\mu_{1}-\omega / 2^{s+2,2 \rho}}(t) \leqq \theta_{2} \rho^{n} \quad \text { for } \quad t \in\left[t_{1}-a(2 \rho)^{\alpha}, t_{1}\right] .
$$

Therefore Lemma 5.4 gives

$$
u(x, t) \leqq \theta_{1}-\frac{\omega}{2^{s+3}} \quad \text { in } Q(\rho)
$$

so that

$$
\omega(\rho) \leqq\left(1-\frac{1}{2^{s+3}}\right) \omega(8 \rho)
$$

This and Lemma 5.6 imply

$$
\omega(\rho) \leqq K \rho^{\lambda}:
$$


If the inequality (5.5) does not hold, then (5.6) is valid and, considering $-u$ instead of $u$, we have (5.20) by the similar argument to the above.

THEOREM 5.2. Let $u$ be a weak solution of the problem (1.1),(1.2). If the boundary value $\psi(x, t)$ belongs to the class $C^{i, \delta / 2}(\partial \Omega)$, then $u$ is Hölder continuous on $\bar{Q}=\bar{\Omega} \times(0, T]$.

The proof is analogous to the proof of the preceding theorem, with the sole difference that Lemma $5.3^{\prime}$ is used instead of Lemma 5.3.

\section{REFERENCES}

[1] D. G. Aronson and J. Serrin: Local behavior of solutions of quasi-linear parabolic equations, Archive for Rational Mechanics and Analysis, 25 (1967), 81-122.

[2] D. G. Aronson and J. Serrin: A maximum principle for non-linear parabolic equations., Annali Scuola Normale Superiore di Pisa, 21 (1967), 291-305.

[ 3 ] O. A. Ladyzenskaya and N. N. Ural'ceva: A boundary value problem for linear and quasi-linear parabolic equations, Dokl. Akad. Nauk. SSSR, 139 (1964), 544547.

[4] J. Serrin: Local behavior of solutions of quasi-linear equations, Acta Math., 111 (1964), 247-302.

[5] G. Stampacchia: Le problème de Dirichlet pour les équations elliptiques de second ordre à coéfficients discontinus, Ann. Inst. Fourier, 15 (1965), 189-258.

Aichi University of Education 